\title{
Specificity of Monoclonal Antibodies against Group B Streptococcus Type II and Inhibition of their Binding by Human Secretions
}

\author{
BARRY M. GRAY, MARIANNE L. EGAN, AND DAVID G. PRITCHARD \\ Departments of Pediatrics and Microbiology, University of Alabama at Birmingham and the Veterans \\ Administration Medical Center, Birmingham, Alabama 35294
}

\begin{abstract}
Mouse monoclonal antibodies against the type-specific polysaccharide antigen of type II group B Streptococcus may be divided into two general groups based on their antigen-binding properties. One group of antibodies binds to both intact and desialylated type II antigen and the binding can be inhibited by $\beta$-galactopyranosides. The second group of antibodies react only with intact type II polysaccharide and are not inhibited by $\beta$ galactopyranosides. The binding of antibodies of the first group can also be inhibited by carbohydrate components of normal human secretions that share structural similarities with the type II polysaccharide. The binding of antibodies of one specificity precluded the binding of antibodies of the other specificity, apparently by competing for binding sites within a combining area that includes both determinants (Pediatr Res 24: 68-72, 1988)
\end{abstract}

Abbreviations

GBS, group B streptococci

TCA, trichloroacetic acid

WGA, wheat germ agglutinin

PBS, phosphate-buffered saline

$\alpha$-MeGal, 1- $O$-Methyl- $\alpha$-D-galactopyranoside

$\beta$-MeGal, 1-O-Methyl- $\beta$-D-galactopyranoside

Type II GBS have attracted considerable attention in recent years because they are the leading type found in adult disease, especially maternal infections (1-3), and are second only to type III GBS as a cause of neonatal bacteremia (1). Extensive epidemiological studies have revealed a remarkably high carriage rate of GBS of all serotypes at delivery for mothers and babies and a relatively low incidence of infections in colonized infants (4). Little is known about the immunological factors that may influence the presence and extent of GBS colonization. Specific antibody, however, is thought to play an important, if poorly understood, role in host defense against actual infections by type II GBS. Inasmuch as it has been shown that antibodies to the type II capsular polysaccharide are protective in mice challenged with an otherwise lethal inoculum of type II GBS (5), the nature of the immune response to the antigen has been the subject of numerous studies (5-10). Lancefield and Freimer (6) first reported that rabbits immunized with GBS type II produced two

Received December 14, 1987; accepted March 3, 1988.

Supported in part by Program Project Grant 5 P01 17812 from the National Institute of Child Health and Human Development, Grant AI 19941 from the National Institute of Allergy and Infectious Diseases, Grant RG 1702 A4 from the National Multiple Sclerosis Society, and by Veterans Administration Medical Research Funds.

Correspondence Barry M. Gray, M.D. University of Alabama at Birmingham, Department of Pediatrics, University Station, Birmingham, AL 35294. general populations of antibodies: one precipitated only with the "complete" antigen prepared by extraction in cold TCA, whereas the other population reacted with either the complete TCA antigen or an "incomplete" antigen prepared by extraction in hot $\mathrm{HCl}$ (6). The $\mathrm{HCl}$ antigen was described as incomplete because the extraction process destroyed a labile determinant, later identified as $\mathrm{N}$-acetylneuraminic (sialic) acid (7). Freimer (8) demonstrated that precipitation of the incomplete antigen could be inhibited by galactose and various $\beta$-galactopyranosides. A possible chemical basis for these immunological findings was suggested by structural studies on the type II polysaccharide by Jennings et al. (11). Unlike the capsular polysaccharides of GBS types Ia, Ib, and III $(12,13)$, the type II polysaccharide was found to possess two distinct single sugar sidechains, a $\beta$-linked galactose and an $\alpha$-linked sialic acid residue. The two general populations of antibodies against the type II polysaccharide observed previously might then be dependent on the presence of one or the other sugar side chain residue.

Polyclonal rabbit antisera have been used extensively in all the early immunochemical studies of type II GBS. The studies of Lancefield and coworkers $(5,6)$ described above used rabbit antisera absorbed with whole cells of various GBS strains and serotypes. Using unabsorbed rabbit antisera, Kasper et al. (10) reported the existence of numerous cross-reactions between the type II polysaccharide and antisera to GBS types $\mathrm{Ia}, \mathrm{Ib}$, and III. It is noteworthy that they did not observe such cross-reactions in human sera after immunization with type II polysaccharide. Uhlenbruck et al. (14) reported that rabbit antisera to type II GBS reacted with several different plant and animal galactans, suggesting the presence of common galactose-containing antigenic determinants in both the galactans and the type II polysaccharide. To more precisely define the nature of the relevant antigenic determinants of the GBS type II polysaccharide, mouse monoclonal antibodies were produced and used in our study.

\section{MATERIALS AND METHODS}

Bacteria. GBS type II, Lancefield strain 18RS21, was grown in Todd-Hewitt broth to logarithmic phase, harvested by centrifugation, washed in PBS $(0.05 \mathrm{M}$ sodium phosphate, $0.1 \mathrm{M}$ $\mathrm{NaCl}, \mathrm{pH} \mathrm{7.4)}$ and heat killed at $80^{\circ} \mathrm{C}$ for $15 \mathrm{~min}$. A vaccine was prepared by resuspending the cells in PBS to an absorbance of 0.6 at $590 \mathrm{~nm}$. $\mathrm{HCl}$ extracts of this strain were prepared for capillary precipitation tests, as previously described (15).

Monoclonal antibodies. BALB/c female mice were immunized intraperitoneally with $0.1 \mathrm{ml}$ of the GBS type II vaccine, with a second immunization 4 wk later. A booster of $0.1 \mathrm{ml}$ intravenously and $0.1 \mathrm{ml}$ intraperitoneally was given 4 days before fusing the spleen cells with $\mathrm{Ag} 8.653$ cells (16). Another mouse was immunized with a mixture of GBS types Ib, II and the nontypable strain, SS1073. Details of hybridoma cloning, screening, isotype identification, and production of antibodies in ascitic 
fluid are described elsewhere (17). Antibody concentrations in ascites fluid were determined by quantitative precipitation with purified type II polysaccharide.

ELISA. Type II polysaccharide was prepared from GBS strain 18RS 21 by mutanolysin extraction and purification over a Sepharose 6B column, essentially as described by De Cueninck et al. (18). Antigen was coupled to poly-L-lysine (19) to facilitate adsorption to the walls of the plastic microtiter wells (Dynatech Laboratories, Alexandria, VA). Coupled antigen was diluted to $1 \mu \mathrm{g} / \mathrm{ml}$ of polysaccharide in PBS and $250 \mu \mathrm{l}$ were dispensed into the wells. Vacant adsorption sites were blocked with $0.1 \%$ bovine serum albumin in PBS. Monoclonal antibodies (ascitic fluid) were diluted in PBS containing 0.05\% Brij-35 (Aldrich Chemical Co., Atlanta, GA). Antibody (with or without the inhibitors described below) was allowed to incubate in the wells for $1.5 \mathrm{~h}$, and unbound material was removed by washing with deionized water. Rabbit anti-mouse immunoglobulin conjugated to horseradish perioxidase (Cooper Biomedical, Malvern, PA) was diluted 1:1000 in PBS-Brij, added to the wells, and incubated for $1 \mathrm{~h}$. After washing out excess conjugate, $o$-phenylenediamine/ $\mathrm{H}_{2} \mathrm{O}_{2}$ substrate was added, color was developed for $15 \mathrm{~min}$, and absorbance measured at $405 \mathrm{~nm}$ in a Titertek Multiskan ELISA Reader.

The basic ELISA procedure was modified slightly to study the ability of various carbohydrates to inhibit the binding of the monoclonal antibodies to bound type II antigen. The glycosides $\beta$-MeGal, $\alpha$-MeGal, and the disaccharide lactose (Sigma Chemical Co., St. Louis, MO) were serially diluted in PBS-Brij and $200 \mu \mathrm{l}$ were added to antigen-coated wells. A constant amount of antibody in a total volume of $50 \mu \mathrm{l}$ was then added to the wells containing different concentrations of the inhibitors. The amount of monoclonal antibody used (approximately $0.5 \mu \mathrm{g} /$ $250 \mu \mathrm{l}$ well) was that found to give absorbance readings high on the straight portion of the ELISA curve, as illustrated on the left in Figure 3. The final sugar concentrations ranged from $0-100$ $\mathrm{mM}$. Antibodies were incubated and processed as described above for the basic ELISA procedure.

The ability of carbohydrate components of human milk and saliva to inhibit in the ELISA for type II antigen was also studied. Human saliva was heated to $100^{\circ} \mathrm{C}$ for $10 \mathrm{~min}$ and centrifuged to remove precipitated proteins and debris. Saliva supernates were diluted in PBS-Brij and $200 \mu \mathrm{l}$ were added to the wells after which $50 \mu \mathrm{l}$ of diluted antibody was added and incubated as above. Human milk was centrifuged at $8000 \times g$ for $30 \mathrm{~min}$ to float the lipids and sediment the cells. The aqueous portion was removed and heated to $100^{\circ} \mathrm{C}$ for $10 \mathrm{~min}$, centrifuged, diluted, and used in the same manner as the saliva. To study oligosaccharide components of human milk other than lactose, a milk sample was clarified by precipitating proteins with $5 \%$ trichloroacetic acid at $0^{\circ} \mathrm{C}$, followed by centrifugation. The supernatant solution was then fractionated on a $1.5 \times 90 \mathrm{~cm}$ column of BioGel P2 eluted with $1 \%$ acetic acid. Fractions were tested for total carbohydrate and sialic acid, and examined by thin-layer chromatography on silica gel 60 plates $(20 \times 20 \mathrm{~cm}, 125 \mu \mathrm{m}$ thickness, Pierce Chemical Co., Rockford, IL) run in 8:3:1 ethyl acetate-pyridine-water. Six fractions contained, respectively, free monsaccharides, lactose, trisaccharides, tetrasaccharides, pentasaccharides, and larger sugars. These fractions were lyophilized, dissolved, $(2 \mathrm{mg} / \mathrm{ml})$ in PBS-Brij, and tested as above for inhibition of the various monoclonal antibodies.

A third set of experiments was carried out to obtain information about the antibody binding sites on the type II polysaccharide. If the side chains are very closely spaced, as the proposed structure suggests (11), it is possible that antibodies of one specificity may interfere with the binding of antibodies of the other specificity. Alternately, it is also possible that due to the three dimensional orientation of the side chains of the polysaccharide, antibodies of the two specificities may bind in an additive manner. These possibilities were studied by measuring the binding of monoclonal antibodies of the two specificities, both separately and in combination. To be sure that the maximum absorbance was not limited by the second antibody concentration, these experiments were repeated with varying dilutions of anti-mouse conjugates $(1: 250$ to $1: 4000)$. Under the ELISA conditions used, there is a more than $40 \%$ excess of conjugate present.

To further assess the competition for binding sites, the inhibitory effect of WGA was tested. WGA is a plant lectin that binds to the sialic acid residues of all the GBS type-specific polysaccharides (20). WGA (Sigma) was diluted in PBS-Brij (0-100 $\mu \mathrm{g}$ in $200-\mu \mathrm{l}$ well) and allowed to incubate in the wells for $1 \mathrm{~h}$ before adding monoclonal antibodies $(50 \mu \mathrm{l}$, approximately $0.5 \mu \mathrm{g}$ / well).

\section{RESULTS}

Ten hybridomas producing monoclonal antibodies against GBS type II were cloned and studied. All were of the $\operatorname{IgM}_{\kappa}$ isotype. The monoclonal antibodies could be divided into distinct groups based on whether or not $\beta-\mathrm{MeGal}$ inhibited their binding to the type II polysaccharide antigen. The binding of eight monoclonal antibodies was inhibited by $\beta$-MeGal whereas the binding of two monoclonal antibodies was not inhibited. This is illustrated in Figure 1 for two representative monoclonal antibodies, F5 and B19.

The addition of $\beta$-galactopyranosides had no effect on the binding of B19 to the type II antigen in the ELISA (Fig. 1, left). Monoclonal antibody F5, however, was almost totally inhibited by as little as $10 \mathrm{mM} \beta$-MeGal (Fig. 1, right). Inhibition by lactose required higher concentrations but was complete at 75 $\mathrm{mM}$. There was no inhibitory effect with $\alpha$-MeGal. Monoclonal antibody D20 exhibited properties very similar to those of F5.

The monoclonal antibodies of the two groups also could be differentiated by their capillary precipitation reactions with purified type II polysaccharide and $\mathrm{HCl}$-extracted (desialylated) type II polysaccharide. The two antibodies whose binding could not be inhibited by $\beta$-galactosides gave precipitin reactions only with the complete type II antigen. The eight monoclonal antibodies that could be inhibited by $\beta$-MeGal reacted with both the complete and the desialylated type II antigens.

Antibodies that could be inhibited by $\beta$-MeGal (D20, F5) also were inhibited by human milk and saliva (Fig. 2). Maximal, essentially complete inhibition was observed with either milk or saliva, at concentrations of $40 \%$ by volume. Monoclonal antibody B19, however, was not inhibited at these concentrations. In additional experiments (not shown) milk oligosaccharide fractions, including trisaccharides and larger oligosaccharides, inhibited the binding of D20 $(1 \mu \mathrm{g} /$ well $)$ by $91-93 \%$, indicating that the inhibitory effect of milk was not due solely to the presence of lactose.

Figure 3 shows the results of experiments carried out to study the nature of the antibody binding sites for the monoclonal antibodies of the two groups. On the left in Figure 3 monoclonal antibodies B19 and D20 were tested at concentrations of $0.02-5$ $\mu \mathrm{g} /$ well. Maximum binding yielded an absorbance of 0.53 in each case. When the same concentration of both B19 and D20 were tested in combination (B19 + D20), the maximum binding was unchanged. This effect was essentially the same at all conjugate dilutions showing that the maximum absorbance was not limited by second antibody concentration. If both monoclonal antibodies were able to bind unhindered to their respective determinants on the polysaccharide, the absorbance obtained in the ELISA procedure would be much greater for the combination of the two antibodies than for either one alone. However, the failure to demonstrate an additive effect of the two antibodies suggests that they compete for the same combining area, despite their distinct differences in specificity. The binding of the two antibodies was partially additive only at lower concentrations, where binding sites were not fully saturated.

To further test the effect of competition for the same combining area, wheat germ agglutinin was allowed to incubate in the ELISA wells before adding the antibodies. Although WGA may 

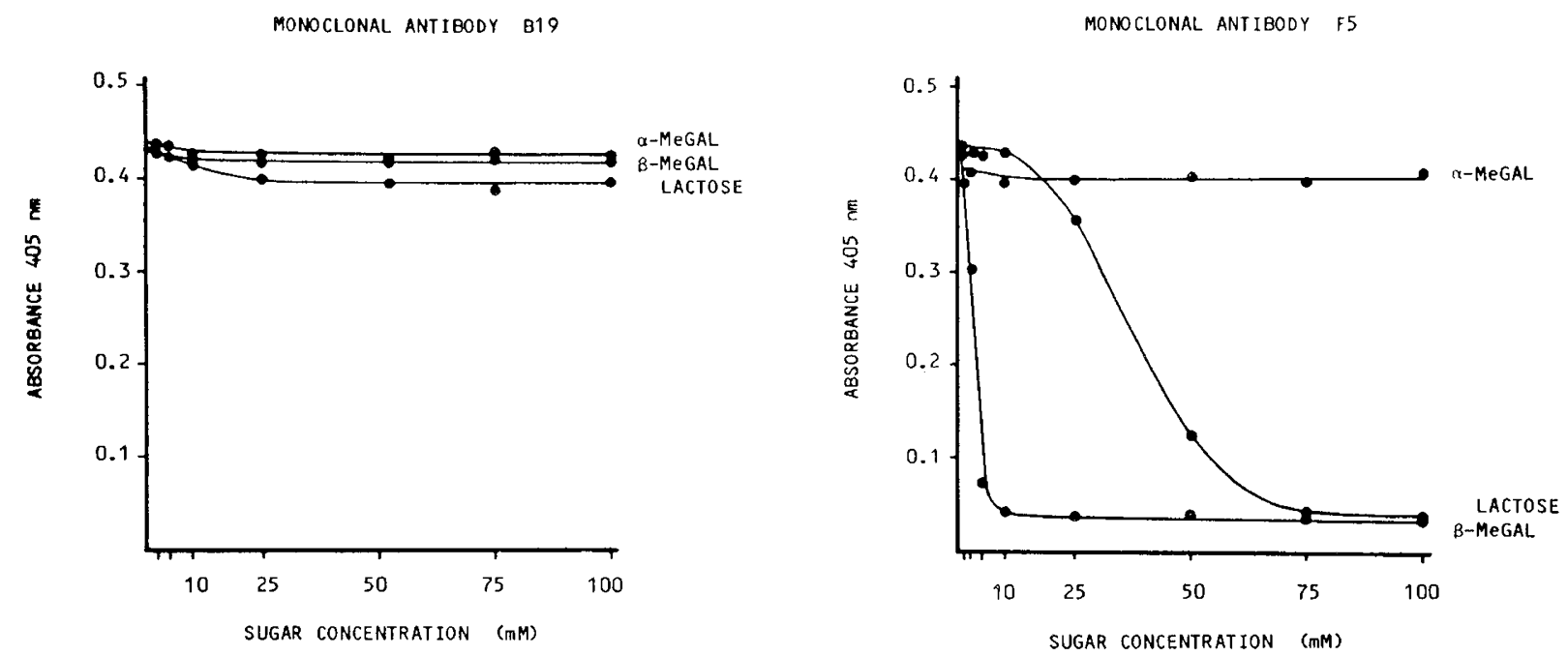

Fig. 1. Results of an ELISA for antibody to GBS type II when constant amounts $(1 \mu \mathrm{g} /$ well) of monoclonal antibody were incubated with increasing concentrations of the indicated sugars. Results obtained with monoclonal antibody B19 are shown on the left and those obtained with monoclonal antibody F5 are shown on the right. Data displayed in the figures are representative of at least five separate experiments.
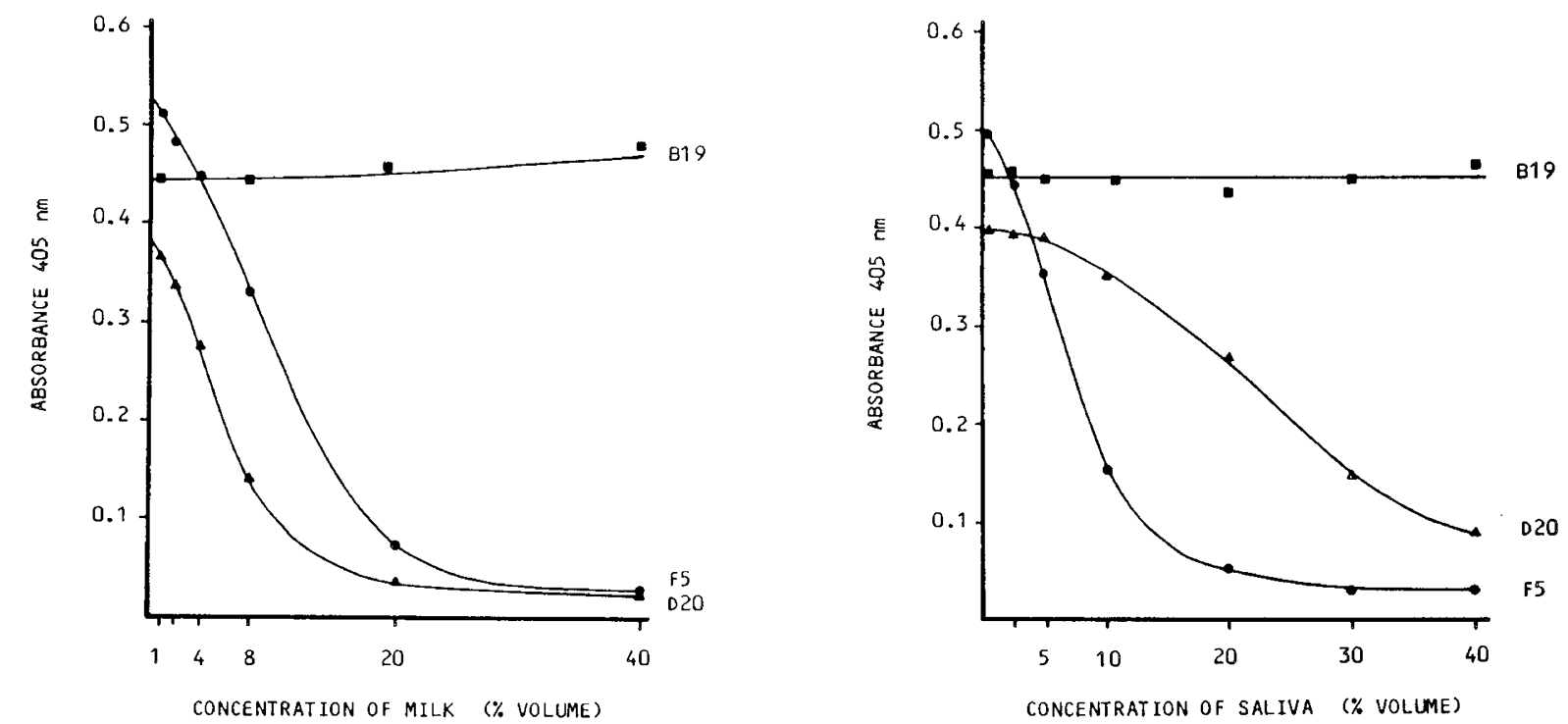

Fig. 2. The effect of increasing concentrations of clarified human milk (left) and saliva (right) on the binding of monoclonal antibodies B19, F5, and D20 to the GBS type II polysaccharide.
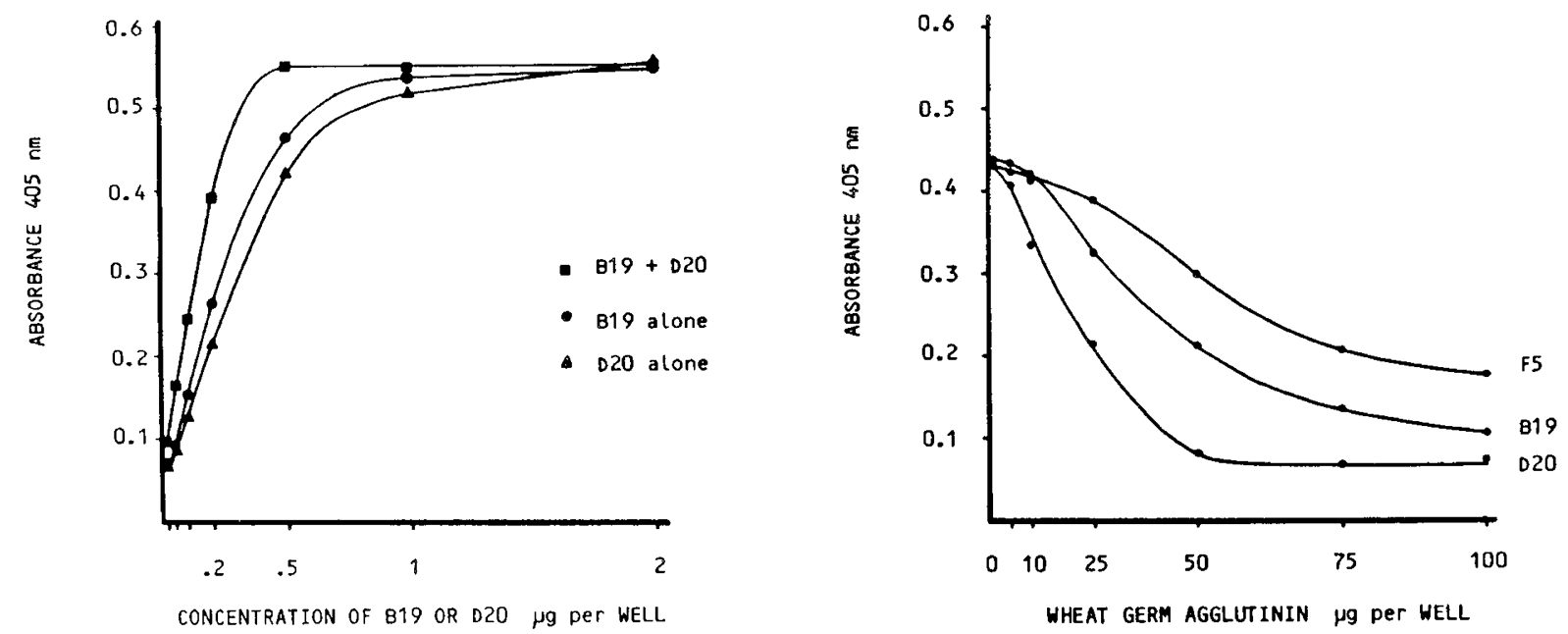

Fig. 3. ELISA results when increasing amounts of monoclonal antibodies B19 and D20 were tested separately or in combination (left). On the right the effect of adding increasing amounts of WGA on the binding of the monoclonal antibodies is shown. 
also bind to antibodies, little inhibition was observed without this preincubation step. As shown on the right in Figure 3, increasing concentrations of WGA resulted in decreasing binding of monoclonal antibodies that could be inhibited by $\beta-\mathrm{MeGal}$ (D20, F5), as well as for those monoclonal antibodies (B19) that are not inhibited by the galactoside.

\section{DISCUSSION}

This study has shown that mouse monoclonal antibodies against the type II GBS polysaccharide may be divided into two distinct categories based on whether or not they can be inhibited by $\beta$-galactosides. It is significant that $\beta$-methylgalactopyranoside strongly inhibited the binding of one group of monoclonal antibodies whereas $\alpha$-methylgalactopyranoside did not inhibit. Compared to $\beta$-MeGal, lactose was a less effective inhibitor on a molar basis, presumably because of the steric hindrance of the glucose residue compared to that of the smaller methyl group.

The ELISA inhibition procedure described herein makes it possible to characterize a particular polyclonal antiserum with respect to its relative content of antibodies of the two specificities. In a separate study (Gray BM, Pritchard DG, Springfield JD, unpublished observations) involving a large number of human and rabbit antisera, the fraction of antibodies to type II GBS whose binding could be inhibited by $\beta$-MeGal varied from 0 to almost $90 \%$.

The chemical structure proposed for the GBS type Il polysaccharide involves two distinct single sugar side chains, a galactose and a sialic acid (11). However, it would be a mistake to think of the two general groups of monoclonal antibodies to the polysaccharide simply as being specific for either a galactose or sialic acid side chain determinant. Antigenic determinants of polysaccharides are known to be considerably larger than single sugar residues. Kabat's (21) classical studies with dextrans and with the type III pneumococcal polysaccharide suggested that antibodies bind optimally to a hexasaccharide portion of the polysaccharide chain. However, the long held idea that antibodies bind preferentially to the nonreducing end-sugars of polysaccharide side chains (21) is no longer universally accepted. Glaudemans (22) argues convincingly that the apparent decrease in immunodominance of side chain sugars from the most exposed inward is an incorrect interpretation of the data (22).

The group of monoclonal antibodies that could not be inhibited by $\beta$-galactosides also could not be inhibited by sialic acid at neutral pH. This was previously shown to be the case for rabbit antibodies to the type II polysaccharide (10). However, removal of the sialic acid residues by mild acid hydrolysis completely destroyed reactivity of the polysaccharide with these monoclonal antibodies.

These studies further show that the monoclonal antibodies of the two general classes compete for binding sites on the antigen despite their very different specificities. In the type II polysaccharide structure proposed by Jennings et al. (11), both single sugar sidechains are linked to galactose residues separated by an $\mathrm{N}$ acetylglucosamine residue in the polymer backbone. The observation that antibody of only one specificity may bind at a time is probably due to the proximity of the two determinants. WGA, which has been shown to bind to the sialic acid residues of the type II polysaccharide (20) also was effective in blocking the binding of either antibody. Although the observed mutually exclusive binding of antibodies of the two specificities to the GBS type II antigen may merely reflect steric hinderance, it is also possible that the binding of one antibody induces a conformational change that interferes with binding by antibody of the other specificity.

It is a new and intriguing finding that human secretions can inhibit the binding of certain antibodies against type II antigen. The inhibitory components of the secretions appear to be car- bohydrates possessing structural similarities with the GBS type II antigen. Lactose and various other galactosyl oligosaccharides of human milk were shown to inhibit. Saliva and vaginal secretions also contain large amounts of galactosyl oligosaccharides bound to mucins that are structurally similar to the GBS polysaccharides $(23,24)$. The observed inhibition by human secretions raises new questions regarding the roles of humoral and mucosal immunity against type II GBS. In this respect it is important to recognize that whereas GBS colonization of mucosal surfaces is extremely common, actual infections are rare (4). Humoral immunity to type II GBS does not appear to prevent colonization of mucosal surfaces by the organism inasmuch as GBS II carriers are far more likely than noncarriers to possess significant levels of serum antibodies to type II GBS (25). The striking structural similarities between the GBS type-specific polysaccharides and components of normal human secretions may actually play a role in facilitating long-term colonization of mucosal surfaces. It is possible that secretory antibodies to type II GBS that cross-react with oligosaccharide side chains of mucin might have the effect of anchoring the bacteria in the mucus layer and inhibiting adherence to epithelial cells. Humoral antibody might then serve to prevent GBS colonization from progressing to serious systemic infections.

Acknowledgment. The expert technical assistance of Mrs. Elizabeth Secor in the production of the monoclonal antibodies is gratefully acknowledged.

\section{REFERENCES}

1. Wilkinson HW 1978 Analysis of group B streptococcal types associated with disease in human infants and adults. Am $\mathbf{J}$ Clin Microbiol 7:175-179

2. Pass MA, Gray, BM, Dillon HC Jr 1982 Puerperal and perinatal infections with group B streptococci. Am J Obstet Gynecol 143:147-152

3. Bayer AS, Chow AW, Anthony BF, Guze LB 1976 Serious infections in adults due to group B streptococci. Am J Med 61:498-503

4. Dillon HC Jr, Khare S, Gray BM 1987 Group B streptococcal carriage and disease: a six-year prospective study. J Pediatrics 110:31-37

5. Lancefield RC, McCarty M, Everly WM 1975 Multiple mouse-protective antibodies directed against group B streptococci: special reference to antibodies effective against protein antigens. J Exp Med 142:165-179

6. Lancefield RC, Freimer EH 1966 Type-specific polysaccharide antigens of group B streptococci. J Hyg (Lond) 64:191-203

7. Lancefield RC, 1972 Cellular antigens of group B streptococci. In: Wannamaker LW, Matsen JM (eds) Streptococci and Streptococcal Diseases. Academic Press, New York, pp 57-65

8. Freimer EH 1967 Type-specific polysaccharide antigens of group B streptococci. II. The chemical basis for serological specificity of the type II $\mathrm{HCl}$ antigen. J Exp Med 125:381-392

9. Baltimore RS, Kasper DL, Baker CJ, Goroff DK 1977 Antigenic specificity of opsonophagocytic antibodies in rabbit antisera to group B streptococci. J Immunol 118:673-678

10. Kasper DL, Baker CJ, Galdes B, Katzenellenbogen E, Jenning HJ 1983 Immunochemical analysis and immunogenicity of the type II group B streptococcal capsular polysaccharide. J Clin Invest 7:260-269

11. Jennings HJ, Rosell KG, Katzenellenbogen E, Kasper DL 1983 Structural determination of the capsular polysaccharide antigen of type II group $B$ streptococcus. J Biol Chem 258:1793-1798

12. Schifferle RE, Jennings HJ, Wessels MR, Katzenellenbogen E, Roy R, Kasper DL 1976 Immunochemical analysis of the types Ia and Ib group B streptococcal polysaccharides. J Immunol 135:4164-4170

13. Wessels MR, Pozsgay V, Kasper DL, Jennings HJ 1987 Structure and immunochemistry of an oligosaccharide repeating unit of the capsular polysaccharide of type III group B streptococcus. J Biol Chem 262:8262-8267

14. Uhlenbruck G, Lutticken R, Bohmer G, Janssen E, Pulverer G, 1985 Group B streptococcus type II antisera have anti-galactan specificities. Zbl Bakt Hyg A $259: 179-187$

15. Dillon HC Jr, Pass MA, Buchanan B 1978 Modified method for the serological identification of group B streptococci. Clin Microbiol 7:599-602

16. Kearney JF, Radbruch A, Liesegang B, Rajewsky K 1979 A new mouse myeloma cell line that has lost immunoglobulin expression but permits the construction of antibody-secreting hybrid cell lines. J Immunol 123:15481550

17. Egan ML, Pritchard DG, Dillon HC Jr, Gray BM 1983 Protection of mice from experimental infection with type III group $B$ streptococcus using monoclonal antibodies. J Exp Med 158:1006-1011

18. De Cueninck BJ, Shockman GD, Swenson RM 1982 Group B type III 
streptococcal cell wall: composition and structural aspects revealed through endo-N-acetylmuramidase-catalyzed hydrolysis. Infect Immun 35:572-582

19. Gray BM 1979 ELISA methodology for polysaccharide antigens; protein coupling of polysaccharides for adsorption to plastic tubes. J Immunol Methods 28:187-192

20. Gray BM, Dillon HC Jr, Pritchard DG 1984 Interaction of group B streptococcal type-specific polysaccharides with wheat germ agglutinin and other lectins. J Immunol Methods 72:269-272

21. Kabat EA 1968 Antigenic determinants and the size of the antibody combining site In: Structural Concepts in Immunology and Immunochemistry. Holt, Rinehart and Winston, New York, pp 80-111
22. Glaudemans CPJ 1986 Immunodominance of terminal sugars revisited. Mol Immunol 23:917-918

23. Feizi T, Gooi HC, Childs RA, Picard JK, Uemura K, Loomes LM, Thorpe SJ, Hounsell EF 1984 Mucin-type glycoproteins. Biochem Soc Trans 12.591596

24. Reddy MS, Levine MJ, Prakobphol A 1985 Oligosaccharide structures of the low-molecular-weight salivary mucin from a normal individual and one with cystic fibrosis J Dent Res 64:33-36

25. Gray BM, Pritchard DG, Dillon Jr HC 1985 Seroepidemiologic studies of group B streptococcus type II. J Infect Dis 151:1073-1080 